\title{
Refractive indices and density of cryovacuum-deposited thin films of methane in the vicinity of the $\alpha-\beta$-transition temperature
}

\author{
A. Drobyshev, A. Aldiyarov, D. Sokolov, and A. Shinbayeva \\ Kazakh National University Almaty 050071, Kazakhstan \\ E-mail: Andrei.Drobyshev@kaznu.kz
}

Received July 28, 2016, revised September 22, 2016, published online April 25, 2017

\begin{abstract}
Experimental studies of the effect of methane condensation temperature on the value of the refractive index and density of the resulting thin films are reported. The main unit of the installation is a high-vacuum chamber, which routinely operates at $10^{-8}-10^{-6}$ Torr. Measurements using a two-beam He-Ne laser interferometer in the vicinity of the methane phase transition temperature $T=20.4 \mathrm{~K}$ in the range of $14-32 \mathrm{~K}$ were carried out. It has been shown that in the vicinity of $T=20 \mathrm{~K}$ the temperature dependence of the refractive index undergoes an abrupt decrease with decreasing temperature. It is assumed that this gap is the result of the phase transition from the orientational disordered phase ( $\alpha$-phase) to the partially ordered phase ( $\beta$-phase) of solid methane. The calculations of the polarizability of the methane molecules in the solid phase at two values of the deposition temperature $T=16 \mathrm{~K}$ and $T=30 \mathrm{~K}$ were performed using the Lorentz-Lorenz equation.
\end{abstract}

PACS: 61.50.-f Structure of bulk crystals;

78.30.-j Infrared and Raman spectra;

68.35.Rh Phase transitions and critical phenomena.

Keywords: methane; thin films, refractive index; density; substrate; phase transition.

\section{Introduction}

Solid methane has for nearly a century attracted the attention of researchers, and this interest continues unabated to the present, for strong and diverse reasons. The starting point of this long history of research was the discovery by Clusius (1929) [1] of the anomalous behavior of the heat capacity of methane at temperature $T=20.4 \mathrm{~K}$. In 1959 James and Keenan [2] demonstrated theoretically that this anomaly may be explained as the phase transition from the high-temperature orientationally disordered phase of methane ( $\alpha$-phase) to the partially ordered phase at a temperature below $T_{c}=20.4 \mathrm{~K}$ ( $\beta$-phase). Later it was discovered [3-5] that the $\beta$-phase is the intermediate state of solid methane towards the full orientation ordering phase ( $\gamma$-phase).

The observed peculiarities in the properties of solid methane, both structural and other (i.e., optical, thermal, mechanical) are largely due to the nuclear spin relaxation processes and their influence on the rotational and translational subsystems of the methane crystal lattice. Study of these processes has been carried out either directly using structural methods $[6,7]$ and the method of nuclear magnetic resonance [8-10], or indirectly, by examining the impact of conversion processes on the macroscopic characteristics of solid methane. This most clearly affects the vibrational spectra of methane in the range of translational and librational vibrations $[11,12]$, as well as the thermophysical properties of methane, such as heat capacity $[13,14]$, thermal conductivity [15], and density [16,17].

This article presents results of the studies of the influence of deposition temperature of methane on the refractive index and density values of the resulting thin films. In contrast to a rather large number of studies of equilibrium solid methane samples [6,16-18], here we demonstrate results obtained directly in the course of low-temperature deposition of the samples, which for this reason were essentially in a non-equilibrium state. Measurements were carried out in the vicinity of the phase transition temperature $T=20.4 \mathrm{~K}$ in the range of $14-32 \mathrm{~K}$.

The transition from the orientationally disordered $\alpha$-phase to the partially ordered $\beta$-phase and back changes the conditions of interaction between the external electromagnetic field and methane molecules, so as to change the contribution of the rotational subsystem to the process. This should affect the value of the refractive index, and the temperature de- 
pendence of this index must undergo a jump in the vicinity of the transformation temperature. In addition, we keep in mind the fact that the mechanism of spin-nuclear conversion in the solid phase of methane are not yet fully understood $[19,20]$. Therefore, a study of the optical and thermal properties of solid methane in non-equilibrium states may contribute to this understanding.

\section{Experimental setup}

The main unit of the experimental setup is a high-vacuum chamber which routinely operates at $10^{-8}-10^{-6}$ Torr (described in detail elsewhere [21]). This pressure was obtained by continuous evacuation with a turbomolecular pump Turbo-V-301 backed with a dry scroll vacuum pump SH-110. Pressure measurement was conducted using a converter FRG-700 with a AGC-100 controller.

Copper substrate covered with planar silver film was used. This substrate with a diameter of $60 \mathrm{~mm}$ was put in thermal contact with a closed-cycle helium GiffordMcMahon refrigerator and placed inside a vacuum chamber. The double-stage cooling system cooled the substrate to $14 \mathrm{~K}$. A resistor heater was connected to the end of the second stage and, with the collaborative work of the refrigerator and heater, the temperature could be varied from $14-200 \mathrm{~K}$. The temperature was monitored by a TS 670-1.4 silicon diode connected to a M335/20 temperature controller, which kept the temperature constant to within $0.5 \mathrm{~K}$.

In this study we used methane gas produced by IHSAN TECHNOGAZ with purity of $99.99 \%$. The gas was injected from a vessel of known volume $V$ through a needle valve. By measuring the change in the pressure in this calibrated volume, we could determine the amount of the gas that was introduced into the chamber:

$$
m=\frac{\Delta P V \mu}{R T}
$$

where $m$ is masse of the introduced methane, $\mathrm{kg} ; \Delta P$ is pressure difference, $\mathrm{Pa}$, in calibrated volume $V \cdot \mathrm{m}^{3}$, before and after gas injection; $\mu$ is masse of methane molecule, mol; $R$ is universal gas constant, $\mathrm{J} / \mathrm{K} \cdot \mathrm{mol} ; T$ is gas temperature, $\mathrm{K}$.

Thanks to special protective screens, all of the injected gas was deposited on the substrate, when the vacuum chamber was closed. The thickness $d$ of the deposited film and its refractive index $n$ were measured using two interference patterns (Fig. 1), generated by two He-Ne lasers and P25a-SS-0-100 photomultiplier tubes. So the density $\rho$ of condensed film of methane could be determine as:

$$
\rho=\frac{m}{S d},
$$

where $S$ is surface area of the condensation, $\mathrm{m}^{2} ; d$ is thickness of the condensed film, $\mathrm{m}$.

The interference curves for each laser beam during the deposition of methane at the temperature $T_{c}=16 \mathrm{~K}$ and

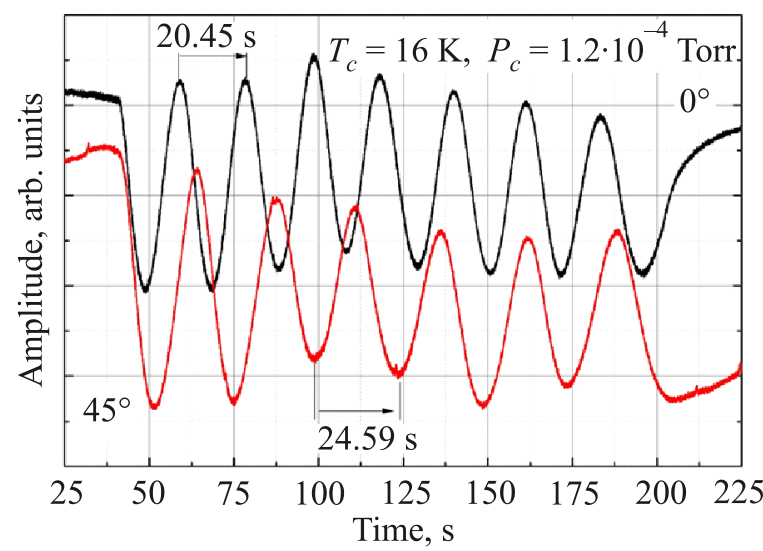

Fig. 1. Interference curves for each laser beam during the deposition of methane at the substrate temperature $T_{c}=16 \mathrm{~K}$ and gas pressure $P_{c}=1.2 \cdot 10^{-4}$ Torr. The upper curve corresponds to the angle of incidence of $0^{\circ}$, the lower curve was obtained at an angle of incidence of $45^{\circ}$.

gas pressure $P_{c}=1.2 \cdot 10^{-4}$ Torr are shown in Fig. 1 . The upper curve corresponds to the angle of incidence of $0^{\circ}$; the lower curve was obtained at an angle of incidence of $45^{\circ}$. Measurements were performed at a frequency of $100 \mathrm{~Hz}$, which makes it possible to determine the period of oscillation to within $\pm 0.05 \mathrm{~s}$.

So in this article we present the results of the experimental studies of the refractive index and the density of thin films of methane, deposited on the metal substrate in the range of $14-32 \mathrm{~K}$. The pressure of deposition was $P_{c}=$ $=1.2 \cdot 10^{-4}$ Torr. The thickness of samples was $d=1.5 \mu \mathrm{m}$. The main sources of error were related to the measurement of the interference period and random error $(0,3 \%)$. Thus, the total error in the measured values of the refractive indices amounts to no more than $0.6 \%$.

\section{Results and discussion}

Before discussing the results, we note once again, that the data shown in Fig. 2 and 3 were obtained at the fixed temperature of the film deposition, and that each data point corresponds to a newly prepared film.

Figure 2 shows the results of our measurements of the refractive index of methane compared to those of other authors. It is evident that our results are in good agreement with the other authors' data, apart from [24,26]. For example, in [17] at $T=30 \mathrm{~K}$ and equilibrium pressure the refractive index of methane was measured to be $n=1.333$, whereas in [22] for the same conditions the value of $n=1.329$ was obtained. In [23] the value of $n=1.323$ was calculated for the temperature $T=20 \mathrm{~K}$. A value of $n=1.350$ obtained for $T=20 \mathrm{~K}$ by approximation of high-temperature data in [24] appears somewhat overstated.

As seen in Fig. 2, our dataset has a distinct gap in the vicinity of the phase transition temperature $T=20.4 \mathrm{~K}$. In our opinion this is due to the fact that each of these datasets is related to different phase states of a thin film of 


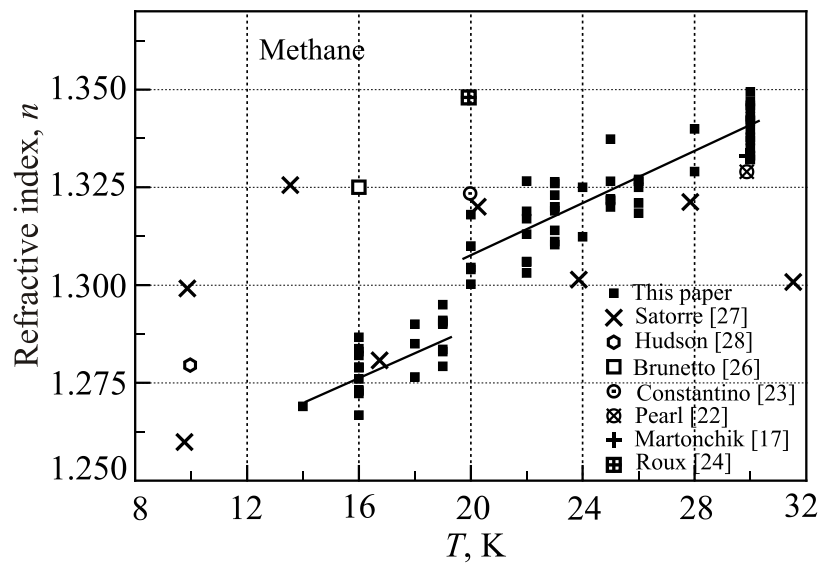

Fig. 2. Plots of refractive index of methane against deposition temperature.

methane. The data in the temperature range $16-19 \mathrm{~K}$ relates to partially orientational ordered $\beta$-phase of methane, while the temperature interval $20.4-30 \mathrm{~K}$ corresponds to the $\alpha$-state with hindered rotating molecule of methane in the crystal lattice sites. Thus it can be concluded that the accompanying partial orientational ordering of the methane molecules upon the $\alpha-\beta$-transition [2] results in a substantial and abrupt decrease of the refractive index of the samples in the vicinity of the phase transition temperature.

As can be seen from Fig. 2, most of the values for $n$ $[17,22,23,26-28]$ are in satisfactory agreement with our data, except for [24]. Possible causes for this deviation are given in [17].

With regard to the reduction of the refractive index with decreasing temperature, common for both phases, this behavior is also characteristic of some other cryofilms. For example, for carbon dioxide [29] the refractive index decreases from $n=1.35$ for the deposition temperature of $T=$ $=77 \mathrm{~K}$ to $n=1.22$ for $T=10 \mathrm{~K}$. Under the same conditions, the refractive index of ammonia [30] decreases from $n=1.41$ to $n=1.37$. The similar behavior was earlier observed in our previous works in the studies of cryovacuum films of carbon dioxide and water [31,32], the results being in good agreement with other data [29,30].

Simultaneously with the measurement of the refractive indices of methane films, their densities were determined depending on the deposition temperature. These data are shown in Fig. 3. The error in measurement of density $\rho$ does not exceed $4-5 \%$ and is determined mainly as the error in measurement of the residual pressure of methane gas in the calibration volume, and the random error.

Comparison of our data with the results of other authors allows us to draw the following conclusions. First, there is a significant difference in the densities of the equilibrium samples $[6,17,18]$ and those obtained directly (as here) during cryodeposition [27]. In particular, in [6] the lattice parameters of solid methane are investigated using $\mathrm{x}$-ray diffraction in the vicinity of the $\alpha-\beta$-transition $(T=20.4 \mathrm{~K})$.

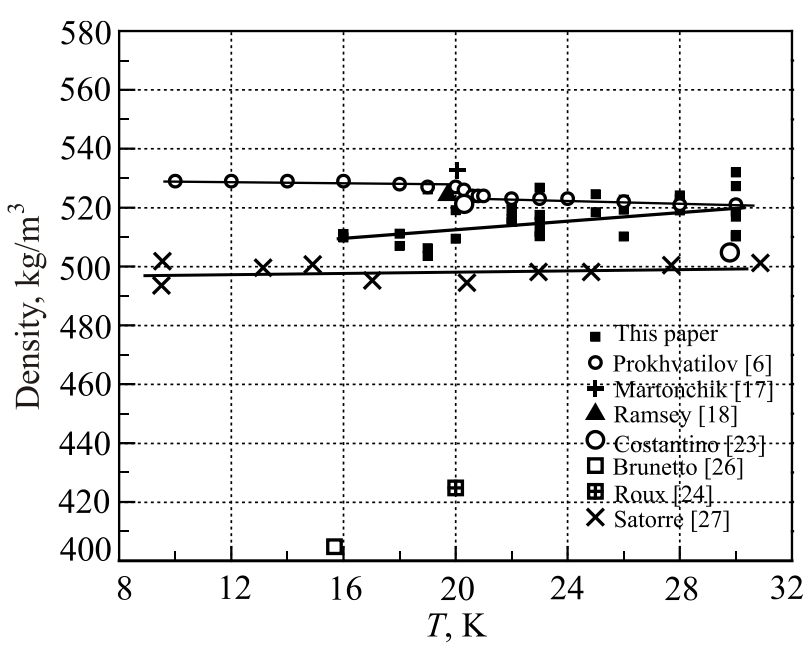

Fig. 3. Relationship between the density of methane cryofilms and their deposition temperature.

The samples were deposited from the gas phase on a substrate at a temperature of $T=5 \mathrm{~K}$ and further annealed for 2 hours at a temperature of $35-40 \mathrm{~K}$. The lattice parameters of a defect-free polycrystalline sample of methane were measured at progressively lower temperatures. In the vicinity of the phase transition temperature $T=20,4 \mathrm{~K}$, an abrupt increase in the density of methane was clearly observed [6].

Analysis of the results of our measurements of the density of methane leads to the following conclusions. As can be seen, the density of the methane films remains practically constant with decreasing deposition temperature. A minor decrease in the density values is slightly higher than the measurement error. Our results are in excellent agreement with the other data [27] obtained in the same manner. The data in $[24,26]$ clearly deviate from the majority of the results. For the data obtained in [24], deviation of the results from those obtained by other authors is characteristic, including the values of the refractive index.

A slight decrease in the density of methane with decreasing deposition temperature may be due to the increasing porosity of the samples. This agrees with the data for other cryodeposited gases [29,30], for which the density of cryofilms is typically reduced as a result of reduction in the deposition temperature. As such, for carbon dioxide, the change in the deposition temperature from 77 to $20 \mathrm{~K}$ leads to a drop in the cryofilms density from $\rho=1670 \mathrm{~kg} / \mathrm{m}^{3}$ (at $77 \mathrm{~K}$ ) to $\rho=1080 \mathrm{~kg} / \mathrm{m}^{3}$ (at $20 \mathrm{~K}$ ). With respect to the density in the vicinity of the phase transition temperature, it should be noted that the error of our measurements of methane density (about 4-5\%) does not allow us to identify the phase transition from the $\alpha$ - to $\beta$-phase.

Available data for the coefficients of refraction and density of methane produced during the same experiment allowed us to determine the polarizability of the methane molecules in the solid phase. These calculations were performed using the Lorentz-Lorenz equation by analogy to 
the calculations of polarizability of carbon dioxide carried out in [29]:

$$
\alpha=\left[\left(n^{2}-1\right) /\left(n^{2}-2\right)\right] 3 M / 4 \pi \rho N_{A},
$$

where: $\alpha$ is polarizability volume, $\mathrm{m}^{3}, M$ is molar mass, $M=16.04 \mathrm{~kg} / \mathrm{kmol} ; N_{A}$ is Avogadro's number, $N_{A}=$ $=6.022 \cdot 10^{26} 1 / \mathrm{kmol} ; n$ is refractive index for $\lambda=632.8$ $\mathrm{nm} ; \rho$ is density of solid methane, $\mathrm{kg} / \mathrm{m}^{3}$.

We have calculated polarizability $\alpha$ at two values of the deposition temperature $T=16 \mathrm{~K}$ and $T=30 \mathrm{~K}$, corresponding to samples in two different states of the rotational subsystem of the crystal lattice of methane. For $T=16 \mathrm{~K}$, we used the measured values of density of $\rho=510 \mathrm{~kg} / \mathrm{m}^{3}$ and refractive index of $n=1.275$. The corresponding value of polarizability was $\alpha_{16 \mathrm{~K}}=(2.15 \pm 0.08) \cdot 10^{-30} \mathrm{~m}^{3}$. For $T=30 \mathrm{~K}$ we used the values of density of $\rho=520 \mathrm{~kg} / \mathrm{m}^{3}$ and refractive index of $n=1.340$; the value of polarizability was calculated to be $\alpha_{30 \mathrm{~K}}=(2.60 \pm 0.08) \cdot 10^{-30} \mathrm{~m}^{3}$.Thus, the calculated relative change in polarizability of methane in this temperature range is $\alpha_{16 \mathrm{~K}} / \alpha_{30 \mathrm{~K}}=0.83$.

\section{Conclusions}

In recent years, laboratory studies of cryodeposited gases including methane have been actively ongoing as part of astrophysical investigations [25-28]. These studies are similar to our research in terms of problem formulation, conditions and methods of measurements. They rightly take into account the importance of simultaneous measurement of refractive index and density of cryofilms in the same experiment, because the values of both of these parameters depend on the conditions of cryodeposition. Results presented here are in good agreement with the publications cited above and can also be interesting and useful from the point of astrophysics. The purpose of our research was a more detailed study of temperature dependence of the refractive index and density of cryofilms solid methane, for which, as noted earlier [2-5], at $T=20.4 \mathrm{~K}$ the transition from the orientational disordered phase ( $\alpha$-phase) to the partially ordered phase ( $\beta$-phase) occurs. It is assumed that this transition must influence the refractive indices of solid methane. As seen in Fig. 2, in the vicinity of $T=20 \mathrm{~K}$, the temperature dependence of refractive index undergoes an abrupt decrease with decreasing temperature, which confirms this assumption.

As for the density of methane cryofilms, our results are in good agreement with the data of authors working on a similar procedure $[25,27]$. The absence of peculiarities in temperature dependence of density in the neighborhood of $T=20 \mathrm{~K}$ may be due to the lack of the necessary experimental precision. Moreover, the increasing sample porosity with decreasing temperature of deposition can compensate for the effect observed in [6]. Thus, we assume that the increase in the density of the crystal due to the decrease of the lattice parameter with temperature decreasing is compensated by increasing of samples porosity degree.

We have calculated the polarizability $\alpha$ at two deposition temperatures of $T=16 \mathrm{~K}$ and $T=30 \mathrm{~K}$, corresponding to samples in two different states of rotational subsystem of crystal lattice of methane. The calculated relative change in polarizability of methane in this temperature range is $\alpha_{16 \mathrm{~K}} / \alpha_{30 \mathrm{~K}}=0.83$. We can assume that observed change in polarizability is the result of transition of a part of the free rotators to an orientationally ordered state. Since the main contribution to methane polarizability brings the rotational subsystem, the decrease in the number of rotators should lead to a decrease of polarizability. In addition the change of polarizability may also be due to the fact $[33,34]$, that the polarizability is proportional to the size of molecule, and that it depends on the rovibrational state, which varies substantially as a result of $\alpha-\beta$-transition [26,35-37]. The decreasing of polarizability, calculated by us, is in good agreement with the results of Costantino and Daniels [23], which presents data on the temperature dependence of dielectric constant of solid methane, the value of which is directly related to polarizability.

This research was supported by the Ministry of Education and Science of the Republic of Kazakhstan, Grant N 3118/GF4-15. Thanks to PhD Tokmoldin Nurlan for discussions during the course of the work and useful comments on this manuscript.

1. K. Clusius, Z. Phys. Chem. 3, 41 (1929).

2. H.M. James and T.A. Keeman, J. Chem. Phys. 31, 12 (1959).

3. J.H. Colwell, E.K. Gill, and J.A. Morrison, J. Chem. Phys. 39, 653 (1973).

4. J A. Kruis, L. Popp, and K. Clusius, Z. Elektrochem. 43, 664 (1937).

5. E. Bartholorne, G. Drikos, and A. Eucken, Z. Phys. Chem. 39, 371 (1938).

6. A. Prokhvatilov and A. Isakina, Fiz. Nizk. Temp. 9, 419 (1983) [Sor. J. Low Temp. Phys. 9, 213 (1983)].

7. D.R. Baer, B.A. Fraase, D.H. Riehl, and R.O. Simmons, J. Chem. Phys. 68, 1411 (1978).

8. A.J. Nijman and N.J. Trappeniers, Physica B 95, 147 (1978).

9. P.A. Beckman, M. Bloom, and I. Ozier, Can. J. Phys. 45, 1712 (1976).

10. S. Buchman, D. Candela, W. Vetterling, and R. Pound, Phys. Rev. B 26, 1459 (1982).

11. C. Chapados and A. Cabana, Can. J. Chem. 50, 3521 (1972).

12. Yuki Miyamoto, Mizuho Fushitani, Daisuke Ando, and Takamasa Momose, J. Chem. Phys. 128, 114502 (2008).

13. M. Bagatskii, D. Mashchenko, and V. Dudkin, Fiz. Nizk. Temp. 33, 728 (2007) [Low Temp. Phys. 33, 553 (2007)].

14. M.I. Bagatskii, V.G. Manzhelii, I.Ya. Minchina, D.A. Mashchenko, and I.A. Gospodarev, J. Low Temp. Phys. 130, 459 (2003).

15. E. Pisarska, P. Stachwiak, and A. Jezowski, Fiz. Nizk. Temp. 33, 768 (2007) [Low Temp. Phys. 33, 587 (2007)]. 
16. V.G. Manzhelii and A.M. Tolkachev, Sov. Phys. Solid State 5, 2506 (1964).

17. J. Martonchik and G. Orton, Appl. Opt. 33, 8306 (1994).

18. W.H. Ramsey, Roy. Astr. Soc. 5, 471 (1963).

19. A.J. Nijman and A.J. Berlinsky, Phys. Rev. Lett. 38, 408 (1977).

20. A.J. Nijman and A.J. Berlinsky, Can. J. Phys. 58, 1049 (1980).

21. A.S. Drobyshev and T.A. Prokhodtseva, J. Low Temp. Phys. 119, 431 (2000).

22. J. Pearl, N. Ngoh, M. Ospina, and R. Khanna, J. Geophys. Res. 96, 477 (1991).

23. M.S. Costantino and W.B. Daniels, J. Chem. Phys. 62, 764 (1975).

24. J.A. Roux, B.E. Wood, A.M. Smith, and R.R. Plyler, Arnold Engineering Development Center Tech. Rep. AEDC- TR-7981 (1979); NTIS accession number AD A088269

25. M. Bouilloud, N. Fray, Y. Benilan, H. Cottin, M.C. Gazeau, and A. Jolly, Monthly Notices Roy. Astronom. Soc. 451, 08 (2015).

26. R. Brunetto, G. Caniglia, G.A. Baratta, and M.E. Palumbo, Astr. Phys. J. 686, 1480 (2008).

27. M.A. Satorre, M. Domingo, C. Millan, R. Luna, R. Vilaplana, and C. Santonja, Planetary, Space Sci. 56, 1748 (2008).
28. Perry A. Gerakines and Reggie L. Hudson, Astrophys. J. Lett. 805, L20 (2015).

29. M. Domingo, R. Luna, M.A. Satorre, C. Santonja, and C. Millán, J. Low Temp. Phys. 181, 1 (2015).

30. B.E. Wood and J.A. Roux, J. Opt. Soc. Am. 72, 6 (1982).

31. A.S. Drobyshev and D.N. Garipogly, Fiz. Nizk. Temp. 22, 814 (1996) [Low Temp. Phys. 22, 584 (1996)].

32. A.S. Drobyshev, N.V. Aatapina, D.N. Garipodly, S.L. Maximov, and E.A. Samyshkin, Fiz. Nizk. Temp. 19, 567 (1993) [Low Temp. Phys. 19, 404 (1993)].

33. T. Guella, Thomas M. Miller, J.A.D. Stockdale, B. Bederson, and L. Vušković, J. Chem. Phys. 94, 6857 (1991).

34. T.M. Miller, in: CRC Handbook of Chemistry and Physics, CRC Press/Taylor and Francis, Boca Raton, FL (2009).

35. Masaaki Miki and Takamasa Momose, Fiz. Nizk. Temp. 26, 899 (2000) [Low Temp. Phys. 26, 625 (2000)].

36. Franklin H. Frayer and George E. Ewing, J. Chem. Phys. 46, 1994 (1967).

37. C. Chapados and A. Cabana, Can. J. Chem. 50, 3521 (1972). 